\title{
Informações em saúde: a importância dos inquéritos populacionais
}

\author{
Health information: the relevance of health surveys
}

Francisco Viacava 1

\footnotetext{
1 Departamento de Informações em Saúde, Centro de Informação Científica e Tecnológica da Fiocruz. Av. Brasil 4.365, 21045-900, Rio de Janeiro RJ, Brasil viacava@cict.fiocruz.br
}

\begin{abstract}
Assuming that health statistics should be considered as an organized data system composed by vital statistics, administrative records, morbidity and mortality data, it is stressed the relevance in generating population based statistics on health status and use of health services as a compensation for the limitations of health services data that are based on users of the health system or vital statistics not always associated with social variables. Health surveys are the only way to generate health information to evaluate the health system performance and the monitoring of social inequalities in health and in the use of health services. It is pointed out some main issues concerning the use of health surveys by other countries and the methods employed to build a health statistics system as part of the health sector reform. Some of the possibilities and limitations of the Brazilian health information system are discussed as well.
\end{abstract}

Key words Health information, Health surveys, Health statistics
Resumo Partindo-se do suposto de que as estatísticas de saúde devem compor um conjunto organizado de dados provenientes dos registros civil, da produção de serviços, das bases de dados de morbi-mortalidade, procura-se enfatizar a necessidade de coletar dados sobre saúde e uso de serviços de saúde, que só podem ser gerados por inquéritos populacionais periódicos, complementando dessa forma as deficiências das informações para monitorar e avaliar as condições de saúde e o desempenho do sistema de saúde brasileiro. Faz-se uma apresentação dos inquéritos desenvolvidos em alguns paises, seus enfoques principais e as limitações mais gerais. Finalmente, apresentase a produção mais recente de documentos e revisões internacionais sobre os mecanismos que vêm sendo utilizados em outros países para a formulação de uma política de informação em saúde como parte da agenda da reforma do setor saúde, que poderiam de certa forma apontar para alguns caminhos no caso brasileiro. Palavras-chave Informação em saúde, Inquéritos populacionais em saúde, Estatísticas de saúde 
$\mathrm{Na}$ passagem para o século 21, muitos países têm organizado discussões sobre o papel das estatísticas de saúde e as necessidades de reformulação de suas bases para procurar responder questões fundamentais sobre suas possibilidades em subsidiar o monitoramento das condições da saúde e o desempenho do sistema de saúde (NRC, 2001; OMS, 1996; OCED, 2001).

No Brasil, as estatísticas de saúde de registro contínuo podem ser agrupadas em quatro grandes áreas: as estatísticas vitais produzidas pelo IBGE; as estatísticas de produção de serviços ambulatoriais e hospitalares que são coletadas pelas secretarias de Saúde e posteriormente consolidadas no Sistema de Informações Hospitalares (SIH), no Sistema de Informações Ambulatoriais (SIA), e no Sistema de Informações sobre Atenção Básica (SIAB), pelo Departamento de Informática do SUS-Datasus; as estatísticas na área de vigilância epidemiológica e monitoramento da situação de saúde, organizadas no Sistema de Agravos de Notificação (Sinan), no Sistema de Nascidos Vivos (Sinasc), e no Sistema de Informações sobre Mortalidade (SIM), que são gerenciados pelo Centro Nacional de Epidemiologia da Fundação Nacional de Saúde (Cenepi/FNS; e as estatísticas relacionadas aos recursos públicos e orçamento do sistema de saúde, em nível municipal, estadual e federal, coordenadas pela Secretaria de Investimentos do MS, que atualmente estão estruturadas no Sistema de Orçamentos Públicos em Saúde (Siops). Além disso, compõem o conjunto das estatísticas de interesse para a área da saúde as pesquisas especiais do IBGE que incluem a pesquisa Assistência Médica Sanitária (AMS), que contém informações sobre a rede de estabelecimentos de saúde desde 1934, com alguns anos de interrupção na década de 1990; os suplementos de Saúde da Pesquisa Nacional por Amostra de Domicílios (PNAD) de 1981, 1986, 1988 e 1998; a Pesquisa Nacional sobre Saúde e Nutrição (PNSN) de 1989; a Pesquisa sobre Padrão de Vida (PPV) de 1996/97; e as Pesquisas de Orçamento Familiar (POF). Estas últimas, limitadas às capitais dos estados, têm assumido importância crescente, na medida que o gasto em saúde feito diretamente pelas pessoas vem adquirindo cada vez maior importância na pauta de consumo das famílias. Outras pesquisas de amplitude nacional que devem ser lembradas são as Demographic and Health Survey (DHS), levadas a campo em 1986, 1991 e 1996, pela Benfam a partir de formulação da Usaid. Na DHS de 1996 foram coletados dados sobre caracte- rísticas dos domicílios, antropometria, saúde reprodutiva, cuidado pré-natal, práticas de contracepção, amamentação, imunização das crianças, conhecimentos sobre AIDS e questões comportamentais relacionadas ao ambiente e consumo de tabaco.

Apesar de se constituírem nas fontes mais fidedignas de informação, o SIM e o Sinasc apresentam limitações importantes no que se refere aos diferenciais de subnotificação ao longo do país, que chegavam em 1995 a 48\% na região Norte, acompanhados por percentual excessivamente alto de óbitos por causas mal definidas (Szwarcwald et al., 1997; Mota e Carvalho, 1999). Por outro lado, o Sinasc, cujo registro parte da Declaração de Nascimento (DN) fornecida pelos hospitais, ou pela Secretaria de Saúde, quando o nascimento ocorre fora de estabelecimento de saúde, ainda não está totalmente implantado em todo o país. Segundo a Rede Interagencial de Informações para a Saúde (RIPSA), em 2001, apenas sete estados brasileiros apresentam regularidade e cobertura igual ou superior a $80 \%$ no SIM e cobertura de $90 \%$ ou mais no Sinasc. A cobertura dos sistemas de informação que compõem o Sinam também é deficiente e a magnitude da subnotificação varia segundo a natureza das doenças e a capacidade de diagnóstico e de notificação dos serviços.

Apesar de serem importantes e amplamente divulgados pelo Datasus, os dados do SIH relativos à produção de serviços hospitalares apresentam uma séria limitação na medida que se referem apenas à produção da rede pública e da rede privada contratada pelo SUS, não contendo, portanto, informações sobre o segmento do setor privado não contratado pelo SUS. A ausência de universalidade da cobertura do sistema de informações hospitalares faz com que o cálculo das taxas de internação refira-se apenas ao SUS. Como a oferta do segmento privado não contratado pelo SUS varia de forma expressiva entre os estados da federação, a cobertura deste sistema também varia. Além disso, apesar dos avanços desenvolvidos pelo Datasus no sentido de se poder contar com dados relativos a cada internação, o problema de reinternação ainda persiste. Isso ocorre porque não é possível identificar, ao longo de um período determinado, as internações relativas à mesma pessoa, no mesmo hospital ou em outros para onde é encaminhada. Esse não é um problema menor, porque altas taxas de reinternação podem ser reflexo de problemas relacionados à 
qualidade do cuidado. Além disso, sabe-se que em qualquer sistema de saúde existem subgrupos populacionais que têm taxas de reinternação superiores à média, envolvendo, em geral, a realização de procedimentos de maior complexidade, sendo responsáveis por grande parte do gasto hospitalar (Roos, 1989). No caso brasileiro, essa limitação do SIH só será contornada depois da implantação do Cartão Nacional de Saúde, por meio do qual será possível ter acesso a todo tipo de serviços utilizados por cada pessoa. Por outro lado, ainda não existem perspectivas de solução definitiva para o problema da universalidade da cobertura do SIH.

A realização de inquéritos, como a PNAD/ 98, com periodicidade garantida, poderia contornar a subestimação das taxas de internação, porém não seria a estratégia adequada para colher informações clínicas necessárias à avaliação da qualidade do cuidado. Por outro lado, a grande vantagem das bases de dados de serviços, como o SIH, é que elas fornecem informação diagnóstica. Em alguns países, as bases de dados de produção de serviços registram o diagnóstico principal, geralmente referido ao motivo principal daquela internação, e os diagnósticos secundários, isto é, as co-morbidades e complicações; os procedimentos diagnósticos e terapêuticos realizados; e outros dados sobre o cuidado. Essas informações constituem-se em importante fonte de dados sobre a morbidade hospitalar e para a análise da qualidade do cuidado. Entretanto, no caso do SIH a ausência de campos para múltiplos diagnósticos e os limitados mecanismos de controle da qualidade dessas informações são problemas que, associados à impossibilidade de identificar a múltipla contagem decorrente de internações de um mesmo paciente, permanecem como graves deficiências para a utilização do sistema, seja para o monitoramento da morbidade hospitalar, seja para a avaliação da qualidade do cuidado.

No caso dos dados ambulatoriais registrados no SIA, as limitações para seu uso são ainda maiores, porque a entrada de dados feita pelas secretarias de Saúde nem sequer é individualizada. Além disso, como no caso do SIH, os dados também não se referem ao universo dos atendimentos ambulatoriais, e nesse caso, o componente SUS é muito menor do que o segmento privado, onde se incluem todos os consultórios médicos privados. No caso do SIA, as informações só podem ser analisadas a partir do volume total atendido em cada estabelecimento ou município. Com a implantação do Cartão
Nacional de Saúde será possível individualizar os atendimentos, pelo menos segundo idade, sexo e local de residência do usuário.

As análises dessas bases de dados de registro contínuo têm focalizado principalmente as mudanças nas tendências no comportamento do perfil de morbi-mortalidade ao longo do tempo e mais recentemente na sua distribuição espacial. Uma limitação comum a todos os dados de produção de serviços, e que em grau menor também se aplica aos sistemas de mortalidade, nascidos vivos e de agravos de notificação compulsória, é a carência de variáveis sociais que, mesmo quando existem, têm baixa confiabilidade e preenchimento incompleto. Embora já exista legislação (portaria 3.947/GM, de 1998) sugerindo que os sistemas de informação devem incluir dados sobre condições sociais, pouco se avançou até o momento nessa direção (Travassos et al., 2000). Entretanto, do ponto de vista da reforma do sistema de saúde, é cada vez mais premente a necessidade de estimular o desenvolvimento de análises sobre as desigualdades sociais relacionadas às condições de saúde, ao acesso e utilização dos serviços e ao financiamento do sistema de saúde, o que implica dois mecanismos importantes: melhorar a qualidade das informações relativas às variáveis sociais nas bases de dados de registro contínuo, e aprofundar as experiências dos inquéritos de base populacional que associam os dados de saúde a condições de vida e trabalho da população.

Neste trabalho, pretende-se traçar um panorama dos inquéritos populacionais sobre condições de saúde e uso de serviços de saúde em diversos países, e apontar para questões envolvidas com a organização e gerenciamento de um sistema nacional de informações que articule os diversos tipos de bases de dados incluindo as estatísticas vitais, os dados sobre produção de serviços, e os inquéritos de base populacional. A análise mais aprofundada de vários aspectos será objeto dos diversos artigos que compõem este número temático de Ciência e Saúde Coletiva.

\section{Inquéritos populacionais de saúde}

Desenvolvidos nos países industrializados desde a década de 1960, os inquéritos populacionais contínuos são instrumentos utilizados para a formulação e avaliação das políticas públicas.

Partindo do princípio de que somente por meio de inquéritos de saúde é possível coletar dados para construir indicadores associados à 
saúde e não apenas às doenças, assim como sobre os fatores de risco e os determinantes sociais do processo saúde/doença, a regional européia da OMS desenvolveu, ao longo da década de 1990, o projeto Eurohis, voltado à formulação de instrumentos e métodos comuns para serem aplicados em inquéritos de saúde na Europa. Tais instrumentos e métodos constituemse numa forma rápida de unir dados originados por diferentes iniciativas sobre as mesmas pessoas ou domicílios, sendo bastante eficazes para identificar subgrupos populacionais e caracterizar sua situação de saúde. Também podem ser considerados meios rápidos, com custo benefício razoável para coleta de dados importantes para a formulação/avaliação de políticas de saúde (Nossikov \& Gudex, 2000).

Nos Estados Unidos, o National Health Interview Survey (NHIS) e o National Health and Nutrition Examination Survey (NHANES) são dois exemplos de inquéritos de saúde que vão a campo anualmente com metodologias e objetivos distintos. O NHIS, realizado pela primeira vez em 1957, coleta informações auto-referidas sobre a ocorrência anual de doenças agudas e acidentes, existência de doenças crônicas e incapacidades, e utilização de serviços de saúde. Atualmente a amostra inclui 40.000 domicílios e cerca de 102.000 indivíduos. Quanto ao NHANES, que foi a campo pela primeira vez em 1971, os dados são coletados pelo exame físico das pessoas incluindo medidas bioquímicas e fisiológicas, o que torna mais objetiva a caracterização do estado de saúde, e evita os problemas relacionados com a morbidade referida (Rice, 2001).

Mais recentemente, a cooperação entre o National Center for Health Statistics (NCHS) e o Agency for Health Care Research and Quality (AHRQ) gerou o Medical Expenditure Panel Survey (MEPS), estruturado sobre quatro componentes: domicílios, nursing homes, provedores de serviços (hospitais, médicos e empresas de home care) e planos de saúde. No caso deste último componente, as informações sobre o tipo de seguro são colhidas no componente domiciliar e também por meio de empresas públicas e privadas, permitindo avaliar a participação dos locais de trabalho no custo do seguro saúde. O MEPS, na verdade, substituiu pesquisas mais antigas como o National Medical Care Expenditure Survey (NMCES), que foi a campo de 1977 a 1987. Além disso, nos Estados Unidos existem vários inquéritos de base populacional específicos como o National Immunization Sur- vey (NIH), o National Household Survey on Drug Abuse (NHSDA) e o National Health Care Survey (NHCS), entre outros (Rice, 2001).

Uma avaliação feita sobre o sistema de estatísticas de saúde dos Estados Unidos identificou vários problemas, tais como a superposição e duplicação de dados e, conseqüentemente, ineficiência na produção de informações; o acesso muito restrito aos dados gerados por serviços de saúde do setor privado; a fragmentação da coleta de dados e a falta de padronização de terminologias e definições das variáveis, que comprometem a possibilidade de comparação de dados de diferentes fontes e a comunicação entre as bases de dados; deficiências na disseminação das informações aos profissionais de saúde; falta de uma política mais geral que defina procedimentos de coleta e disseminação de informações; pouca capacidade das bases de dados para gerar informações para o nível local ou para subgrupos populacionais, e a baixa capacidade dos sistemas de informações para permitir um acompanhamento longitudinal com uma visão integrada dos processos de prevenção e tratamento de doenças e os resultados nos níveis de saúde da população (NRC, 2001). Atualmente, algumas iniciativas vêm sendo tomadas no sentido de associar as informações do NHIS com o MEPS e com o HANES, o que garantirá uma melhor qualidade das análises sobre as condições de saúde, a prevalência de doenças crônicas, a cobertura dos serviços de saúde e uso e gasto com utilização de serviços (Rice, 2001).

$\mathrm{Na}$ Inglaterra, ainda que os censos demográficos decenais incluam informações sobre saúde, o que significa poder fazer estimativas até para pequenas áreas, o monitoramento das condições de saúde, de comportamentos prejudiciais à qualidade de vida e do uso de serviços de saúde da população é feito através de inquéritos contínuos, sendo os mais importantes o General Health Survey (GHS), que vai a campo desde 1971, e o Health Survey for England (HSE), implantado em 1993. Existem ainda numerosas pesquisas que foram a campo nos anos 90, dirigidas para problemas específicos de saúde (Sturgis et al., 2001).

No Canadá, o National Population Health Survey (NPHS), cujos dados já foram coletados no biênio 1994-1995 e posteriormente em 19981999, é um inquérito que, com uma amostra longitudinal, vai a campo a cada dois anos, cobrindo cerca de 17 mil domicílios. Encontra-se atualmente em campo (quinto ciclo) e tem da- 
dos sobre condições de saúde, uso de serviços de saúde, condições de trabalho e hábitos de vida que influenciam as condições de saúde. Além desse inquérito de abrangência nacional, existem também diversas iniciativas desenvolvidas ao nível das províncias (CIHI, 2001).

Do ponto de vista de instrumentos desenvolvidos por agências internacionais, o mais recente é o World Health Survey (WHS), que vem sendo formulado e aplicado pela OMS em alguns países, numa tentativa de conseguir superar a falta de dados existentes principalmente nos países mais pobres, o que tem sido um complicador na aplicação da metodologia preconizada pela OMS para Avaliação de Desempenho de Sistemas de Saúde (Braveman et al., 2000; Almeida et al., 2001).

Nos países em desenvolvimento, geralmente os inquéritos populacionais em saúde têm um caráter mais episódico e são baseados em modelos desenvolvidos por organismos internacionais como o Banco Mundial.

As pesquisas domiciliares foram implantadas na América Latina por um acordo entre a United States Agency for International Development (Usaid) e o Interamerican Statistical Institute (Iasi), a partir do ideário do Plano Atlântida elaborado pelo Departamento de Comércio do governo americano, em associação com o US Bureau of the Census, incorporandose as recomendações das Nações Unidas (Ferrer, 2000). O projeto previa criar uma estrutura de informações contínuas reunindo características demográficas básicas e de emprego, com suplementos anuais abordando temas específicos, com periodicidade variável segundo o tema.

O Brasil aderiu à proposta descrita acima com o desenvolvimento da PNAD no segundo trimestre de 1967. Mas, contrariando o que era preconizado - coleta de dados sendo feita quatro vezes ao ano -, a partir de 1971 os dados passaram a ser coletados apenas uma vez ao ano. Além disso, o tamanho da amostra, cuja base inicial era de cerca de 30.000 domicílios, teve de ser ampliado de forma a atender às demandas regionais. Em 1982, a amostra já abrangia 110.000 domicílios e um total de 550.000 pessoas. Os suplementos tiveram seus conteúdos elaborados com os ministérios, que se apóiam em quadros técnicos de institutos e universidades, mas são definidos sem levar em conta critérios técnicos de periodicidade (Fletcher \& Ribeiro, 1988). No caso específico da saúde, os suplementos foram a campo em 1981, 1986 e em 1998.
Em 1989 o IBGE, por meio de convênio com o Instituto Nacional de Alimentação e Nutrição (Inan), realizou a PNSN, que tem uma estrutura muito semelhante às da PNAD e da DHS no que se refere às variáveis demográficas e sociais. Além da caracterização do estado nutricional, também contém dados sobre estado de saúde e consumo de serviços de saúde.

A PPV, realizada em 1996-1997, seguiu um modelo desenvolvido nos anos 80 pelo Banco Mundial, o Living Standard Measurement Study (LSMS), que cobre aspectos relativos a demografia, pobreza, serviços sociais, trabalho, renda, gasto e infra-estrutura de saúde. Cerca de 25 países já realizaram esse tipo de inquérito domiciliar e em muitos deles a pesquisa foi a campo mais de uma vez (Sadana et al., 2000; Ferrer, 2000). No caso da PPV, foram entrevistados 5.000 domicílios representativos apenas das regiões Nordeste e Sudeste, com uma coleta de dados ao longo de 12 meses, entre março de 1996 e março de 1997.

\section{Dimensões cobertas por inquéritos de saúde e consumo de serviços de saúde}

Os inquéritos de saúde cobrem um variado repertório sobre as medidas de saúde e do uso de serviços de saúde. A seguir, apresentam-se alguns aspectos metodológicos sobre as dimensões mais comumente incorporadas nessas pesquisas, que incluem medidas do estado de saúde, da ocorrência de doenças, de fatores de risco, de acesso e uso de serviços de saúde, da cobertura por planos de saúde, e de aspectos demográficos e sociais do informante ou do domicílio (Hupkens CLH et al., 1999; Aday, 1989). Além desses aspectos, quase todos cobertos no Suplemento Saúde da PNAD/98, será feita menção também ao gasto domiciliar privado em saúde, aspecto incluído no referido suplemento. Isso, entretanto, não deve impedir que outras dimensões sejam objetos de investigação nos próximos inquéritos de saúde no Brasil, como saúde mental, acidentes e violência, comportamentos relacionados ao risco de adoecer, estilos saudáveis de vida, acolhimento dos serviços de saúde, e suporte social para onde se reorientam os inquéritos de saúde nos países industrializados. 


\section{Medidas de estado de saúde}

Como parte da Estratégia Global de Saúde para todos, lançada pela OMS em 1979, elegeram-se, na primeira metade da década de 1980 , cerca de 80 indicadores, 21 dos quais considerados passíveis de se obter apenas através de inquéritos de saúde (WHO, 1990). Entre estes, oito indicadores são relacionados com o estado de saúde e incluem: auto-avaliação do estado de saúde, porcentagem da população economicamente ativa empregada portadora de deficiências, número de dias de restrição temporária de atividade por motivo de saúde, por pessoa e por ano, porcentagem de pessoas com diversos níveis de restrição crônica por idade e sexo, Disability Free Life Expectancy (DFLE), prevalência de algumas doenças crônicas, avaliação do nível de interação social, e incapacidade permanente para o trabalho e suporte social. Em 1992, em uma nova consulta técnica feita pela OMS, foram analisados os resultados de 20 inquéritos e identificados como os indicadores mais freqüentemente utilizados: a auto-avaliação do estado de saúde, o número de dias de restrição temporária por motivo de saúde e a porcentagem de pessoas com diferentes graus de incapacidade de longa duração (WHO, 1992).

A auto-avaliação do estado de saúde é um dado facilmente coletado, a respeito do qual existem estudos que encontraram taxas de $80 \%$ de concordância entre auto-avaliação do estado de saúde e avaliação clínica da presença ou ausência de condição crônica (Blaxter, 1990). Sturgis et al. (2001) revisaram diversos instrumentos recentemente utilizados na Inglaterra para avaliar o estado de saúde, considerando a validade e a confiabilidade de várias medidas entre as quais a auto-avaliação. Para esses autores, o problema do uso da auto-avaliação para medir o estado de saúde reside na falta de clareza sobre o que se está medindo, já que os determinantes do estado de saúde são múltiplos, incluindo aspectos físicos, cognitivos, emocionais, culturais e socioeconômicos, e a saúde é em si um conceito complexo e multidimensional (NCHS, 1990; Sturgis et al., 2001; Sen, 2002). No entanto, o dado é tradicionalmente coletado, inclusive pelo Censo Demográfico da Inglaterra, com o intuito, muitas vezes, de qualificar as informações mais diretamente relacionadas à limitação de atividades causadas por problemas de saúde de longa duração. Constitui-se, também, em indicador importante sobre o comportamento da população com relação à busca por serviços de saúde, especialmente entre a população mais idosa (Sturgis et al., 2001).

Quanto ao grau de limitação física ou de restrição permanente das atividades rotineiras, alguns inquéritos utilizam-se de escalas que medem o grau de autonomia na realização dessas atividades, como as ADL (Activities of Daily Living), que são entendidas como atividades que as pessoas necessitam desenvolver para sobreviver (Gudex \& Lafortune, 2000). Aí se incluem atividades de autocuidado (banhar-se, vestir-se e alimentar-se), mobilidade (andar, subir escadas, subir e descer da cama), comunicação (capacidade de ver, ouvir e falar). Medidas mais refinadas incluem a avaliação do exercício pleno de tarefas necessárias para viver de forma independente em uma comunidade, como fazer compras, executar o trabalho doméstico, preparar refeições e administrar finanças (IADL Instrumental Activities of Daily Living).

Existem ainda diversas metodologias que produzem um índice do estado de saúde que incorpora várias dimensões, combinando aspectos físicos e cognitivos, como é o caso do Short Form 36 (SF36), o mais conhecido e validado instrumento desse tipo usado nos países do Primeiro Mundo (Sturgis et al., 2001). Desenvolvido no final dos anos 80 , foi aplicado em numerosas situações, desde estabelecimentos de saúde até inquéritos populacionais. Sua concepção é baseada na definição de oito dimensões ou domínios obtidos por análise fatorial, que foram validados em várias circunstâncias e apresentam boa sensibilidade (Ware e Donald, 1992). Contudo, quando se trabalha com fatores no lugar das respostas a cada item, perde-se capacidade de utilização dos aspectos reportados em cada item de cada fator e, portanto, perde-se a capacidade de interpretação dos resultados. Mas, de maneira geral, acredita-se que o SF36 tenha boa sensibilidade e sua estrutura dimensional ofereça boa representatividade da situação de saúde. Outra qualidade desse instrumento é a eliminação do problema de distribuição excessiva nas pontas da escala, que vai de "excelente" a "muito ruim" (Sturgis et al., 2001). Em 1996 o SF36 foi incorporado ao Health Survey of England (HSE) como questionário auto-aplicável. Um estudo de validação desse instrumento para a Nova Zelândia verificou que a estrutura fatorial foi muito similar para os neozelandeses de origem européia, mas que o mesmo não se verificou para a população maori acima dos 45 anos, quando apenas um fator foi evidenciado, sugerindo que, 
nesse caso, o uso do SF36 não é adequado à visão de saúde dos maoris, que não distingue os aspectos físicos dos mentais (Scott et al., 2001).

Numa revisão de 30 inquéritos sobre medidas de limitação física ou restrição permanente de 23 países da Organization for Economic Cooperation and Development (OECD), verificou-se que os questionários se diferenciaram quanto ao número de itens empregados para mensurar as várias dimensões. Como exemplo temos o EuroQol - sistema descritivo do estado de saúde que incorpora cinco dimensões, e produz um escore de cada estado de saúde -, no qual se incluem apenas duas perguntas sobre atividades rotineiras, contrastando com o SF36, que tem dez perguntas sobre esta mesma dimensão. A prevalência de limitação física de atividades obtida pelos inquéritos depende do número de perguntas, ou seja, quanto mais atividades estiverem sendo medidas, maior será o número de pessoas identificadas como portadoras de deficiências. Outro problema relacionado à avaliação da limitação de atividade diz respeito à subjetividade da informação sobre o nível de gravidade da limitação. Problemas adicionais referem-se à decisão de incluir nas perguntas a possibilidade de realizar as atividades com algum tipo de ajuda ou não; por exemplo, o uso de bengala, óculos ou acompanhantes. Dada a baixa freqüência de incapacidades nas faixas etárias mais jovens, questiona-se também a faixa de idade a partir da qual perguntas sobre limitação de atividade devem ser aplicadas (Bowling, 1994; Gudex \& Lafortune, 2000).

A restrição temporária de atividades por motivo de saúde é uma informação normalmente coletada pelo NHIS e outros inquéritos (NCHS, 1995) e, nesse caso, refere-se ao período de duas semanas, captando, portanto, a presença de episódios mórbidos agudos ou a agudização de problemas crônicos. O período de duas semanas pode variar segundo o instrumento, sendo que alguns preferem estipular uma semana determinada, outros discriminam o tipo de atividade prejudicada (afazeres domésticos, dias de escola, dias de trabalho, etc.). A pergunta mais geral é seguida da informação sobre o número de dias de restrição de atividades, que pode ser associado à pessoa ou ao problema de saúde, já que um dia de restrição pode ser devido a mais de um problema de saúde (Collins, 1997). As respostas individuais são multiplicadas por 26, somadas e divididas pelo número de respondentes. Em outros casos, coleta-se também a informação sobre os dias que a pessoa permaneceu acamada, o que estaria representando restrição temporária de caráter mais severo (WHO, 1996). Além de ser um proxy da morbidade, essa medida é usada para avaliar o impacto das doenças agudas na sociedade, em termos de tempo perdido por motivo de saúde (WHO, 1996).

No que concerne à referência a doenças crônicas, há diversas questões envolvidas. A primeira delas relaciona-se com o fato de, em algumas das doenças crônicas, a pessoa só reconhecer sua doença a partir de um diagnóstico médico. Por esse motivo, os inquéritos tendem a subestimar a prevalência de tais enfermidades. Em outros problemas crônicos, como a dor lombar, por exemplo, que pode ser autoavaliada, a tendência dos inquéritos é superestimar sua prevalência (WHO, 1990). Alguns inquéritos geram informações a partir da manifestação da doença ou sintoma ao longo da vida das pessoas e outros limitam a informação sobre a presença da doença aos últimos 12 meses, sendo este último considerado pela OMS o mais adequado para o monitoramento da saúde da população (WHO, 1990). Embora a OMS sugira que os países devam selecionar doenças de maior impacto em cada realidade, em 1990 definiu-se um pequeno grupo de doenças crônicas que deveriam ser objeto dos inquéritos: hipertensão, asma, bronquite, problemas com tireóide, diabetes, doença crônica de pele, doença crônica de coração, cistite crônica, problema dental crônico, dor nas costas crônica, artrite e acidente vascular cerebral (WHO, 1990). O mesmo documento sugere que câncer não deve ser objeto dos inquéritos populacionais, devendo-se utilizar os dados dos registros de câncer existentes em grande parte dos países. Outra restrição diz respeito à subestimação dos casos de doenças crônicas que implicam internação de longa permanência (senilidade, algumas doenças mentais), já que os inquéritos domiciliares referem-se normalmente à população civil não institucionalizada.

Na revisão realizada em 2000 pela OMS a partir de 18 inquéritos, houve a recomendação de redução do número de doenças crônicas e de estímulo à obtenção de informação sobre doença crônica com base na existência de um diagnóstico médico, e se questionou se a referência à existência de doença deveria ser associada ao período de observação de 12 meses, recomendado anteriormente (WHO, 2000). 


\section{Utilização de serviços de saúde}

Freqüentemente os inquéritos de saúde incluem módulos referentes ao acesso e utilização de serviços de saúde, gerando informações fundamentais para a análise do acesso e utilização de serviços de saúde por áreas geográficas segundo características demográficas, sociais e de saúde. O estado de saúde ou o perfil de necessidades de saúde é um importante determinante da procura por serviços de saúde. A análise do perfil de uso dos serviços de saúde de diferentes grupos populacionais implica que as taxas de utilização sejam controladas pelo estado de saúde e padronizadas por idade e sexo. Entretanto, Black et al. (1999) ressaltam que, apesar do estado de saúde ser um elemento fundamental para a busca de serviços, existe uma inter-relação entre o estado de saúde e a percepção do estado de saúde, que é influenciada, por sua vez, pela oferta de serviços e pelas práticas de atenção à saúde.

Existem dois tipos de dados que são mais comumente coletados. O primeiro diz respeito ao volume de uso de serviços de saúde num período de mais longo prazo, em geral de 12 meses, como nos Estados Unidos (NCHS, 1995), ou 90 dias, como na Dinamarca (Danish Health and Morbidity Survey, 1994). A partir daí, são gerados indicadores do tipo número de consultas médicas, ou consultas odontológicas anuais para determinados grupos populacionais. Em alguns casos, os inquéritos também informam se os serviços utilizados são regularmente os mesmos, sendo este dado considerado também uma expressão do acesso. O segundo tipo de dado coletado considera a utilização mais recente de serviços de saúde, ocorrida, em geral, nos últimos 15 ou 30 dias. Nos Estados Unidos, o NHIS vem procurando ampliar o período de referência relativo às perguntas de acesso e utilização, pois a baixa freqüência dos eventos faz com que as análises de dados gerados a partir da utilização nos últimos 15 dias só possam ser realizadas em níveis mais agregados de observação. No caso das internações hospitalares, considera-se um período maior de observação, usualmente de 12 meses, por ser um evento mais raro e menos facilmente esquecido.

Em alguns inquéritos, as questões relacionadas à utilização de serviços são respondidas apenas pelas pessoas que referem a presença de doença crônica ou de alguma limitação de atividade física. Este foi o caso encontrado em $75 \%$ de 13 questionários latino-americanos ana- lisados por Ferrer (2000). No caso da PNAD/98, todas as pessoas responderam aos quesitos de uso independentemente do estado de saúde.

Nos Estados Unidos, a taxa de utilização de serviços, nos 15 dias que antecederam a entrevista, oscila em torno de 15\% (NCHS, 1995). Na Inglaterra, em 1998, uma taxa muito semelhante foi obtida pelo GHS (ONS, 1998): 12\% dos homens e $17 \%$ das mulheres consultaram um médico generalista nos 15 dias que antecederam a entrevista. No caso do Brasil, os dados da PNAD/98 apontaram um taxa de utilização semelhante às anteriores no caso das mulheres $(15,4 \%)$, enquanto se observou, para os homens, uma taxa mais baixa $(9,9 \%)$ (Viacava $e t$ al., 2001).

A porcentagem de pessoas que referiram internação nos 12 meses que antecederam a entrevista manteve-se estável no período 19821998, permanecendo em torno de 9 a 10\% (UK, DOH, 2000). Na PNAD/98, observou-se uma taxa de internação ao longo de 12 meses de 5,1\% para homens e 8,7\% para mulheres (incluindo parto) (Viacava et al., 2001).

Além desses dados mais gerais que caracterizam o uso de serviços de saúde, e são fundamentais para planejar a organização da oferta, os inquéritos costumam coletar dados relativos à freqüência com que diferentes tipos de serviços são procurados, os motivos da procura, os tipos de atendimento recebido, motivo do não atendimento, tempo de espera para ser atendido, grau de satisfação dos pacientes, etc.

Do ponto de vista da organização da oferta e da análise dos custos envolvidos com a atenção à saúde, embora a doença aguda que envolve restrição de atividades rotineiras seja um fator fundamental para explicar a procura e a utilização por serviços de saúde, o volume de pessoas que procura serviços de saúde sem que essa procura esteja relacionada a um episódio agudo é muito maior (Travassos et al., 2000; Viacava et al., 2001). Assim, por exemplo, na PNAD/98, 55,5\% dos homens e 59,4\% das mulheres que apresentaram restrição de atividade rotineira por motivo de saúde procuraram os serviços de saúde; já entre as pessoas que não apresentaram restrição de atividade, apenas 7,2\% dos homens e $12,1 \%$ das mulheres procuraram serviços de saúde. Entretanto, como somente uma pequena parcela da população apresentou restrição de atividade nos 15 dias que antecederam a entrevista, em termos absolutos isso significa que 5,7 milhões de pessoas no primeiro caso e cerca de 14,7 milhões no segundo ca- 
so procuraram serviços, o que demonstra a importância crescente do consumo de serviços de natureza preventiva ou de acompanhamento da situação de saúde (Viacava et al., 2001).

\section{Gasto direto em saúde}

Um elemento que só pode ser colhido através de inquéritos populacionais é o gasto familiar ou individual com atenção médica (gasto privado), sendo importante distinguir o que foi pago como prêmio às operadoras e o montante diretamente recolhido pelos provedores de serviços. Grande parte dos países procura utilizar informações de provedores e de inquéritos domiciliares para estimar gastos em saúde e também as desigualdades existentes em cada sociedade quanto ao financiamento dos serviços de saúde.

No Brasil esse dado é normalmente captado pelas Pesquisas de Orçamento Familiar (POF), por meio de metodologia que leva em conta a freqüência e o período de observação necessário para coletar informações sobre o gasto domiciliar com cada item da pauta de consumo das famílias. No caso da saúde existem itens, como medicamentos, cujo período de observação corresponde aos últimos 30 dias, e itens com períodos de observação de 90 dias. Os dados publicados geralmente se referem ao impacto relativo desses gastos na pauta anual de consumo. Os dados da POF, obtidos apenas para as capitais do país, são coletados através de registro em cadernetas deixadas nos domicílios por cerca de uma semana.

$\mathrm{Na}$ PPV e no suplemento saúde da PNAD/ 98 também foram coletados dados sobre o gasto em saúde efetuado pelas famílias, porém as informações foram obtidas no momento da entrevista e, eventualmente, os entrevistadores retornaram ao domicílio para completar os formulários, o que significa que a confiabilidade das informações é muito mais baixa do que no caso da POF.

\section{Cobertura por plano de saúde}

Embora menos freqüentemente incluída nos inquéritos de saúde, a cobertura por seguro privado assume cada vez mais importância, dada a necessidade nos países do Primeiro Mundo de se monitorar a situação das pessoas que não têm cobertura seja por plano de saúde público ou privado. Nos Estados Unidos a porcentagem da população nessa situação é da ordem de $14,2 \%$, chegando a $18 \%$ entre adultos de 18 a 64 anos. Por outro lado, $72 \%$ da população maior de 64 anos tem plano privado de saúde (NCHS, 2002). A cobertura é menor em grupos populacionais como as mulheres, principalmente na faixa etária de 55 a 65 anos, que têm $20 \%$ mais chances de não serem cobertas por seguro do que os homens (Lambrew, 2001). No caso brasileiro, dados da PNAD/98 divulgados pelo IBGE indicam que, apesar da cobertura universal do SUS, $25 \%$ da população é coberta por plano de saúde, sendo cerca de $60 \%$ dos planos de saúde financiados integral ou parcialmente pelo empregador (IBGE, 2000).

\section{Limitações dos inquéritos populacionais}

\section{Nível de agregação}

Embora em países mais ricos existam inquéritos em níveis subnacionais (estados ou municípios), a grande parte dos inquéritos de saúde é representativa do nível nacional, já que o tamanho amostral não permite desagregações para níveis geográficos menores. Todos os fóruns de discussão têm enfatizado a necessidade de refazer os desenhos amostrais de modo a incluir a representatividade para níveis geográficos menos abrangentes (NRC, 2001). No caso da PNAD/98, as informações podem ser desagregadas apenas até o nível de Estados.

A impossibilidade de desagregar os dados dificulta muitas vezes a análise do acesso e uso de serviços de saúde, assim como de seus determinantes em grupos populacionais específicos como os idosos, mulheres chefes de família, etc. Por vezes, a única alternativa é a realização de inquéritos de base populacional dirigidas para unidades geográficas menores.

\section{Não resposta}

Um dos problemas dos inquéritos domiciliares são as não respostas que podem resultar de recusa ou de problemas operacionais na coleta. Quando respondentes e não respondentes apresentarem características distintas, as análises provenientes desses dados apresentarão erros sistemáticos.

A imputação de dados é uma alternativa para reduzir o número de casos sem informação, desde que a variável seja importante, que a 
quantidade de casos ignorados não seja maior do que $10 \%$ e que os custos envolvidos sejam compensatórios (Aday, 1989). A premissa básica é que a "imputação" introduza menos erro do que a simples exclusão dos casos. Dados podem ser "imputados" por diversos processos, sendo mais comum fazê-lo a partir de médias gerais ou de subamostras, seleção aleatória de respostas de outros indivíduos, que podem ser definidos como semelhantes segundo outras características de variáveis. Um outro procedimento consiste em ordenar os registros e usar os valores mais próximos como substitutos; finalmente, também é possível fazer estimativas a partir de técnicas estatísticas de regressão (Aday, 1989).

\section{Informantes secundários}

Embora alguns desenhos de inquéritos domiciliares utilizem a amostragem intradomiciliar para selecionar indivíduos que serão entrevistados, é comum a prática de se coletar dados sobre pessoas ausentes por intermédio de informações prestadas por outros moradores, principalmente mulheres e cônjuges dos chefes de família. Isso evidentemente introduz um erro, que é tanto mais importante quanto maior o grau de subjetividade dos quesitos. Nos Estados Unidos, o NHIS é um inquérito que também possui essa característica. Entretanto, para diminuir a freqüência do uso de informantes secundários, os domicílios sorteados são procurados várias vezes até que se encontre pelo menos uma pessoa e através desse contato, procura-se marcar a visita em um momento em que todos os componentes da família estejam no domicílio. De qualquer forma, o informante secundário é usado, amiúde, para pessoas menores de 19 anos e também para as demais pessoas ausentes (NCHS, 1990). No caso da PNAD, os dados sobre as crianças pequenas foram fornecidos preferencialmente pela mãe. Considerando-se a população maior de 14 anos, $48,7 \%$ das pessoas na região urbana e $57 \%$ na região rural avaliaram seu próprio estado de saúde. Entretanto, essa proporção é muito diferenciada entre sexos, sendo que na região urbana $61,3 \%$ das mulheres e $34,6 \%$ dos homens foram os informantes de seu próprio estado de saúde, e na região rural essas porcentagens corresponderam a $69,6 \%$ e $45,3 \%$ respectivamente (Viacava et al., 2001).

\section{Privacidade das informações}

Considerando-se as necessidades mais comuns de utilização das estatísticas de saúde, na grande maioria dos casos, os dados podem ser disponibilizados em formatos agregados, que impedem a identificação das pessoas. Entretanto, havendo necessidade de acesso aos dados individuais e identificados, é preciso dispor de mecanismos que garantam a confidencialidade das informações de indivíduos e grupos, protegendo-as das ações de seguradores, empregadores e até mesmo evitando que se constituam em provas de processo administrativo fiscal ou judicial (Kanaan, 2000). Essa questão, que diz respeito a qualquer base de dados, assume importância nos inquéritos populacionais, porque a probabilidade de recusa depende do grau de confiança da população informante na confidencialidade e no uso da informação que está sendo fornecida.

\section{Tratamento dos dados}

Os dados dos inquéritos populacionais originados em geral de amostras probabilísticas necessitam de tratamento estatístico adequado, já que as estimativas dos parâmetros populacionais são baseadas no tipo de desenho amostral. Sua divulgação precisa ser acompanhada de informações necessárias para que as análises sejam feitas levando em conta os diversos tipos de erros associados ao processo amostral. Adicionalmente, ainda que as informações sobre o processo amostral estejam disponíveis, a sua incorporação na análise estatística dos dados primários não é uma tarefa trivial.

\section{Criação de uma instância de formulação da política de informações}

Apesar de termos concentrado a discussão das estatísticas de saúde ao redor dos inquéritos populacionais, é importante que se reafirme a necessidade da integração e complementaridade das estatísticas de saúde, principalmente do ponto de vista de sua análise e gestão. No caso brasileiro muito já se alertou sobre a fragmentação dos sistemas de informação, cujas lógicas distintas e limitações são decorrentes das próprias razões de seu desenvolvimento (Moraes, 1994).

Como já se mencionou anteriormente, isso também caracteriza a situação existente em outros países. Nos Estados Unidos, em 1998, deu- 
se início a um processo de consulta nacional sobre a política setorial, promovido pelo $\mathrm{Na}$ tional Committee for Vital and Health Statistics (NCVHS), órgão encarregado de assessorar o Department of Health and Human Services (DHHS). Os resultados apontaram para a relevância de se prever (1) quais seriam as necessidades de informação para o próximo século; (2) sob que marco conceitual ela poderia ser organizada para alcançar maior significado; (3) quais os procedimentos mais adequados para coleta, armazenamento e comunicação para garantir a informação de alta qualidade; e (4) como proteger a segurança, a confidencialidade e a privacidade das informações para que ela pudesse ser usada de forma segura na promoção da saúde da população (NCVHS, 2000). Ao longo de três anos de discussão produziu-se um documento contendo recomendações mais dirigidas ao redirecionamento do sistema de estatísticas do que sobre a organização e função, procurando inovar no sentido da coleta dos dados e de sua transformação em informação de utilidade para a área da saúde através da cooperação entre as diferentes agências.

O documento propõe a criação de uma câmara de planejamento para informações em saúde (Health Information Planning Board) de amplo alcance e semi-independente (sua composição não incluiria apenas o setor público), com autoridade e responsabilidade para decidir o quadro geral, a direcionalidade e a coordenação do sistema nacional de dados de saúde, envolvendo parcerias entre provedores de saúde, operadoras de planos de saúde, usuários do setor público e do setor privado, e produtores de dados de saúde.

No caso da Austrália, foi assinado um acordo - National Health Information Agreement - em 1993, prorrogado até 2003, entre as duas principais agências governamentais, o Australian Bureau of Statistics (ABS) e o Australian Institute for Health and Welfare (AIHW), e as autoridades responsáveis pela saúde no nível central, nos estados e territórios, com vistas à adoção de mecanismos necessários à melhoria da coleta, qualidade e disseminação das informações de saúde (Zelmer et al., 1999). A ABS se responsabiliza pela compilação das estatísticas de mortalidade, pela rede privada de atenção e pela condução dos inquéritos domiciliares, inclusive os inquéritos de saúde que vão regularmente a campo. Cabe à AIHW a responsabilidade pela identificação e atendimento às necessidades formuladas pelo governo, pela dissemi- nação dos dados, desenvolvimento de padrões para garantir a comparação dos dados dentro do país e fazer recomendações sobre prevenção, tratamento e promoção de saúde.

No Canadá, foi criado, em 1993, o Canadian Institute for Health Information (CIHI), uma instituição independente, não lucrativa, que tem o mandato de coordenar o desenvolvimento e a manutenção de um sistema nacional integrado de informações de saúde, além de coletar dados sobre serviços de saúde, recursos humanos e gasto em saúde. O sistema preocupa-se com o desenvolvimento de padrões em nível nacional em associação com duas outras instituições: o Health Canadá, responsável pela área de vigilância; e o Statistics Canada, que responde pelas estatísticas vitais, pelo inquérito nacional de saúde, registro de câncer e outras pesquisas geradoras de informação sobre estado de saúde e determinantes de saúde (Zelmer et al., 1999).

$\mathrm{Na}$ Inglaterra, a reforma do National Health Service (NHS) incluiu uma estratégia de informação envolvendo um bilhão de libras para o período 1998-2005, dirigida para a difusão do conhecimento médico atualizado para profissionais e para usuários do sistema. A estratégia está voltada para a implantação do prontuário eletrônico e da NHSnet, que, através de um conjunto de tecnologias, promoverá o acesso imediato do público aos serviços de saúde. Será criada uma biblioteca em Saúde contendo as informações clínicas baseadas em evidências e literatura dirigida aos pacientes. Atualmente, do ponto de vista do arcabouço institucional, o grande provedor das estatísticas oficiais é o Government Statistical Service (GSS), em que estão localizados o Department of Health e o Office for National Statistics (ONS), este último criado em 1996 através da fusão do Central Statistics Office e da Office of Population Censuses and Surveys (OPCS). O ONS responde por todas as estatísticas de saúde da Inglaterra e do País de Gales.

Os exemplos acima citados, apesar de se referirem a poucos países selecionados, demonstram a crescente preocupação dos países em organizar o sistema de estatísticas para melhorar a qualidade da gestão e o monitoramento da situação de saúde.

No Brasil, apesar de o artigo 47 da lei 8.080, de 1990, que cria o Sistema Único de Saúde, mencionar que no prazo de dois anos deveria ser constituído o Sistema Nacional de Informações em Saúde, até hoje esse sistema não foi regulamentado. Uma iniciativa importante to- 
mada em 1996 pela portaria no 820/GM foi a criação da Rede Integrada de Informações para a Saúde (Ripsa), atualmente denominada Rede Interagencial de Informações para a Saúde, que congrega representantes de diferentes instituições no âmbito do Ministério da Saúde e fora dele. Ao reunir profissionais de diferentes instituições produtoras e usuárias de informações em saúde, a Ripsa tem exercido, como principal atividade, a promoção da articulação institucional para a formulação e desenvolvimento de padrões dos indicadores básicos de saúde (IDB), que vêm sendo disponibilizados todos os anos desde 1998. Sua inserção no Ministério da Saúde, inicialmente na Secretaria de Políticas e de Avaliação e hoje no Cenepi, não permite que a Ripsa atue como um fórum de formulação da política de informação em saúde. As parcerias dos diversos setores do MS com o IBGE, Ipea e instituições acadêmicas, embora funcionem a contento no sentido de garantir a qualidade dos indicadores demográficos e socioeconômicos, não envolvem mecanismos formais que garantam a continuidade das ações. Os representantes das instituições produtoras das informações fora do âmbito do MS atuam como assessores, tendo ainda a responsabilidade de fornecer os indicadores correspondentes à sua área de atuação. Além disso, a Ripsa tem sido a instância por meio da qual comitês temáticos se discutem diversas questões relacionadas às informações em saúde. É o caso do geoprocessamento, em relação ao qual a questão é, novamente, a definição de padrões a serem usados em todo o território nacional.

No caso específico dos inquéritos de saúde, as decisões sobre o desenho dos instrumentos são tomadas em outras instâncias do Ministério da Saúde, sem nenhuma articulação com a
Ripsa, de forma episódica e sem nenhum tipo de planejamento ou consideração de ordem técnica a respeito das prioridades e da periodicidade com que as informações deveriam estar disponíveis para a formulação da política de saúde. Ainda que seja um recurso de baixo custo, a utilização da PNAD para efeitos de coleta de informações sobre acesso e utilização de serviços de saúde implica a seleção de um conjunto bastante restrito de informações. Assim, tais informações devem ser cuidadosamente selecionadas, para evitar aumento exagerado do tempo de aplicação do questionário, o que poderia comprometer toda a PNAD. As outras alternativas, financiadas pelo Banco Mundial, como a PPV, apresentam um custo elevado e, nesse caso, opta-se por diminuir o tamanho da amostra que deixa de ser representativa do país.

Dados da PNAD/98 revelam que apenas $50 \%$ dos atendimentos de saúde, nas duas semanas de observação, foram feitos através do SUS, o que significa uma necessidade urgente de realização periódica de inquéritos nacionais de saúde que não se restrinjam às poucas questões inseridas nas PNADs, mas que permitam um efetivo monitoramento do desempenho do sistema de saúde brasileiro público e privado. Por outro lado, para ganhar muito mais domínio no desenvolvimento de análise dos dados com vistas à formulação da política de saúde, é preciso investir na análise das informações em saúde não apenas no nível local, mas também no plano nacional. Isso envolve a concepção de mecanismos para reforço da cooperação e das parcerias já existentes entre as instâncias do MS, os órgãos responsáveis pelas estatísticas de saúde e as instituições acadêmicas, procurando identificar as barreiras existentes e as formas de contorná-las.

\section{Agradecimentos}

Agradeço as valiosas sugestões de Cláudia Travassos, e as contribuições de Mônica Martins, Célia Leitão Ramos, Sílvia Porto e Lígia Bahia. Um agradecimento especial a Cecília Minayo, a idealizadora deste número temático. 
Siglas usadas no texto

\begin{tabular}{|c|c|c|}
\hline ABS & Australian Bureau of Statistics & (Austrália) \\
\hline ADL & Activities of Daily Living & \\
\hline AHRQ & Agency for Health Care Research and Quality & (EUA) \\
\hline AIHW & Australian Institute for Health and Welfare & (Austrália) \\
\hline AMS & Pesquisa Assistência Médico Sanitária & \\
\hline BENFAM & Sociedade Civil Bem-Estar Familiar no Brasil & \\
\hline CENEPI & Centro Nacional de Epidemiologia & \\
\hline CIHI & Canadian Institute for Health Information & (Canadá) \\
\hline $\mathrm{CSO}$ & Central Statistics Office & (Inglaterra) \\
\hline DFLE & Disability Free Life Expectancy & (OMS) \\
\hline DHHS & Department of Health and Human Services & (EUA) \\
\hline DHS & Demographic and Health Survey & (USAID/Brasil) \\
\hline $\mathrm{DN}$ & Declaração de Nascimento & \\
\hline FNS & Fundação Nacional de Saúde & \\
\hline GHS & General Health Survey & (Inglaterra) \\
\hline GSS & Government Statistical Service & (Inglaterra) \\
\hline $\mathrm{HC}$ & Health Canada & (Canadá) \\
\hline HMS & Health and Morbidity Survey & (Dinamarca) \\
\hline HSE & Health Survey for England & (Inglaterra) \\
\hline IASI & Interamerican Statistical Institute & \\
\hline IBGE & Instituto Brasileiro de Geografia e Estatística & \\
\hline INAN & Instituto Nacional de Alimentação e Nutrição & \\
\hline IPEA & Instituto de Pesquisa Econômica Aplicada & \\
\hline LSMS & Living Standard Measurement Study & (Banco Mundial) \\
\hline MEPS & Medical Expenditure Panel Survey & (EUA) \\
\hline MS & Ministério da Saúde & \\
\hline NCHS & National Center for Health Statistics & (EUA) \\
\hline NCVHS & National Committee for Vital and Health Statistics & (EUA) \\
\hline NHANES & National Health and Nutrition Examination Survey & (EUA) \\
\hline NHCS & National Health Care Survey & (EUA) \\
\hline NHIS & National Health Interview Survey & (EUA) \\
\hline NHSDA & National Household Survey on Drug Abuse & (EUA) \\
\hline $\mathrm{NIH}$ & National Immunization Survey & (EUA) \\
\hline NMCES & National Medical Care Expenditure Survey & (EUA) \\
\hline NPHC & National Population Health Survey & (Canadá) \\
\hline NRC & National Research Council & (EUA) \\
\hline OECD & Organization for Economic Cooperation and Development & (Europa) \\
\hline OMS & Organização Mundial da Saúde & \\
\hline ONS & Office for National Statistics & (Inglaterra) \\
\hline OPCS & Office of Population Censuses and Surveys & (Inglaterra) \\
\hline PNAD & Pesquisa Nacional por Amostra de Domicílio & \\
\hline PNSN & Pesquisa Nacional sobre Saúde e Nutrição & \\
\hline POF & Pesquisa de Orçamento Familiar & \\
\hline PPV & Pesquisa sobre Padrão de Vida & \\
\hline RIPSA & Rede Interagencial de Informações para a Saúde & \\
\hline SC & Statistics Canada & (Canadá) \\
\hline SF & Short Form & (Europa/América do Norte) \\
\hline SIA & Sistema de Informações Ambulatoriais & \\
\hline SIAB & Sistema de Informações sobre Atenção Básica & \\
\hline SIH & Sistema de Informações Hospitalares & \\
\hline SIM & Sistema de Informações sobre Mortalidade & \\
\hline SINAN & Sistema de Informação Nacional de Agravos de Notificação & \\
\hline SINASC & Sistema de Informações sobre Nascidos Vivos & \\
\hline SIOPS & Sistema de Orçamentos Públicos em Saúde & \\
\hline SUS & Sistema Único de Saúde & \\
\hline USAID & United States Agency for International Development & (EUA) \\
\hline WHO & World Health Organization & \\
\hline WHS & World Health Survey & (OMS) \\
\hline
\end{tabular}




\section{Referências bibliográficas}

Aday LA 1989. Designing and conducting health surveys: $a$ comprehensive guide. Jossey-Bass Inc., Publishers. São Francisco, 398pp.

Almeida C et al. 2001. Methodological concerns and recommendations on policy consequences of the World Health Report 2000. The Lancet, 357:1.692-1.697.

Black C, Ross N, Ross L 1999. From Health Statistics to Health Information Systems: a new path for the 21st century. Paper comissioned by the National Committee for Vital and Health Statistics. Disponível em http: //www.ncvhs.hhs.gov/hsvision/visiondocuments.html.

Blaxter M 1990. Health and lifesytles. Routledge Inc., Nova York, 288pp.

Bowling A 1994. La medida de la salud: revision de las escalas de medida de lacalidad de vida. Masson, S/A, Barcelona, 222pp.

Braveman P, Krieger N, Lynch J 2000. Health inequalities and social inequalities in health. Bulletin of the World Health Organization, 78(2):232-234.

Canadian Institute for Health Information (CIHI) 2001. Health care in Canada. Disponível em http://www. cichi.ca.

Collins JG 1997. Prevalence of selected chronic conditions: United States, 1990-92. National Center for Health Statistics. Vital Health Stat. 10(194).

Danish Health and Morbidity Survey 1994. Disponível em http://www.sifolkesundhed.dk/udgivelser/web/ SUSY/teks/index.htm

Ferrer M 2000. Los módulos de salud en las encuestas de hogares de America Latina y el Caribe: un análisis de cuestionarios recientes. PAHO. Serie Informes Técni$\cos$ N. 72.

Fletcher PR \& Ribeiro SC 1988. A educação na estatística nacional, pp. 13-41. In Sawyer DO (org.). PNADs em foco - anos 80. Haydn Pimenta Editor, ABEP.

Gudex C \& Lafortune G 2000. An inventory of health and disability-related surveys in OECD countries. Labour Market and Social Policy. Occasional Papers N. 44, UN, Nova York.

Hupkens CLH,van der Berg J, van der Zee J 1999. National health interview surveys in Europe: an overview. Health Policy 47:145-168.

IBGE 2000. Acesso e utilização de serviços de saúde, Brasil 1998. IBGE, Rio de Janeiro, 96pp.

Kanaan SB 2000. Shaping a vision for $21^{\text {st }}$ century health statistics: Interim Report. Washington. DC.

Lambrew JM, 2001. Diagnosing disparities in health insurance for women: a prescription for change. Disponível em http://www.cmwf.org/programs/insurance/ lambrew_disparities_493.pdf

Moraes IH, 1994. Informações em saúde: da prática fragmentada ao exercício da cidadania. Hucitec-Abrasco, Rio de Janeiro.

Mota E \& Carvalho DM 1999. Sistemas de informação em saúde, pp.505-521. In Rouquayrol MZ e Almeida Filho N (orgs.). Epidemiologia \& saúde. MEDSI Editora Médica e Científica, Rio de Janeiro.

NCHS 1990. Americans assess their health: United States, 1987. National Center for Health Statistics. Vital Health Stat. 10(174).

NCHS 1995. Current Estimates From the National Health Interview Survey, 1994. National Center for Health Statistics. Vital Health Stat. 10(193).
NCHS 2002. Early release of Health Insurance Coverage estimates. Disponível em: http://www.cdc.gov/nchs/ about/major/nhis/released200203.htm

NCHVS 2000. Shaping a vision for $21^{\text {st }}$ century health statistics. Interim report. Disponível em: http://www. ncvhs.hhs.gov/hsvision/Vision21stCentury.pdf

NRC 2001. Toward a Health Statistics System for the 21 st Century: Summary of a Workshop. Committee on National Statistics, Perrin ED, Kalsbeek D Scanlan TM Editors. Division of Behavioral and Social Sciences and Education, National Academy Press, Washington, DC. $81 \mathrm{p}$.

Nossikov A \& Gudex C 2000. Overview of the implementation of EUROHIS. Joint ECE/WHO Expert Meeting on Health Statistics Measurements. Working Paper $\mathrm{N}$ 5. Ottawa, Canadá.

Office of National Statistics (ONS) 1998. Morbidity, general health and use of health services. Technical report. Disponível em http://www.doh.gov.uk/pub/ docs/doh/survey2.pdf

Rice DP 2001. Health Statistics: Past, present and future, pp. 7-30. Apresentado em National Research Council: toward a health statistics system for the 21st century: summary of a workshop. National Academy Press, Washington DC.

Roos NP, Shapiro E \& Tate R 1989. Does a small minority of elderly account for a majority of health care expenditures? A sixteen-year perspective. The Milkbank Quarterly 67(3):347-369.

Sadana R, Mathers CD, Lopez AD, Murray CJL, Iburg K 2000. Comparative analyses of more than 50 household surveys on health status. GPE Discussion Paper Series N. 15. EIP/GPE/EBD. WHO.

Scott KM, Sarfati D, Tobias MI, Haslett J 2001. A challenge to the cross-cultural validity of the SF-36 Health Survey: Maori, Pacific, and New Zealand European Ethnic Groups. Seventh Conference on Health Survey Research Methods. DHHS Publication N. (PHS) 01-1013:91-96. US Department of Health and Human Services, Hyattsville, Maryland.

Sen A 2002. Health: perception versus observation. BMJ.

Sturgis P, Thomas R, Purdon S, Bridgwood A, Dodd T 2001. Comparative review and assessment of key health state measures of the general population. Department of Health, Londres.

Szwarcwald CL, Leal MC, Castilho EA, Andrade CLT 1997. Mortalidade infantil no Brasil: Belíndia ou Bulgária? Cadernos de Saúde Pública, 13(2):503516.

Travassos C et al. 2000. Variáveis sociais nos bancos de dados de interesse para a área da saúde, pp. 35-43. In I Seminário Nacional de Informação em Saúde: o setor saúde no contexto da sociedade da informação. Série Fiocruz: eventos científicos 3, Rio de Janeiro; Fundação Oswaldo Cruz.

UK Department of Health 2000. Morbidity, general health and use of health services. General Household Survey 1998. Disponível em www.doh.gov.uk/pub/ docs/doh/survey2.pdf

Viacava F, Travassos C, Pinheiro RS, Brito A 2001. Gênero e utilização de serviços de saúde no Brasil. Relatório final da pesquisa. Fiocruz, Rio de Janeiro, pp. 108.

Ware JE, Donald CD 1992. The MOS - 36 item Short 
Form Health Survey (SF-36) conceptual framework and item selection. Medical Care, 30 (6):473-483.

WHO 1990. Common methods and instruments for health interview surveys. Report of the second WHO consultation. Voorburg, Holanda. Disponível em http://whqlibdoc.who.int/euro/-1993/EUR_ ICP_HST_124.pdf

WHO 1992. Third consultation to develop common methods and instruments for health interview surveys - report of a WHO meeting. Voorburg, Holanda. Disponível em http://whqlibdoc.who.int/euro/ 1993/EUR_ICP_HST_124.pdf

WHO 1996. Health Interview Surveys: towards international harmonization of methods and instruments. (Editado por A de Bruin, HSJ Picavet e A Nossikov). WHO regional publications. European series N. 58.
WHO 1997. Common methods and instruments for health interview surveys in Europe. Report on the $4^{\text {th }}$ WHO Consultation. Copenhagen, 1997.

WHO 2000. Common methods and instruments for health interview surveys in Europe. Report of the WHO Eurohis Mid-term Review. Copenhagen, 2000.

Zelmer J, Virani S, Alvarez R 1999. Recent developments in health information: an international perspective. Encomendado pelo National Committee for Vital and Health Statistics para a reunião Developing the $21^{\text {st }}$ Century Vision for Health Statistics. Disponível em http://www.ncvhs.hhs.gov/hsvision/visiondocuments. html 